\title{
Application of a Validated Stability-Indicating LC Method for the Simultaneous Estimation of Tapentadol and Its Process-Related Impurities in Bulk and Its Dosage Form
}

\author{
Singaram Kathirvel, ${ }^{1}$ Suggala Venkata Satyanarayana, ${ }^{2}$ and Garikapati Devalarao ${ }^{3}$ \\ ${ }^{1}$ Department of Pharmaceutical Analysis, Hindu College of Pharmacy, Amaravathi Road, Guntur 522002, India \\ ${ }^{2}$ Department of Chemical Engineering, JNTUA College of Engineering, Anantapur 515002, India \\ ${ }^{3}$ KVSR Siddhartha College of Pharmaceutical Sciences, Vijayawada 520008, India \\ Correspondence should be addressed to Garikapati Devalarao; devalarao2007@gmail.com
}

Received 20 June 2012; Revised 31 July 2012; Accepted 31 July 2012

Academic Editor: Lian-Wen Qi

Copyright ( 2013 Singaram Kathirvel et al. This is an open access article distributed under the Creative Commons Attribution License, which permits unrestricted use, distribution, and reproduction in any medium, provided the original work is properly cited.

\begin{abstract}
Described is a first reported, simple, rapid, selective, and isocratic stability-indicating RP-LC method for the quantitative determination of tapentadol and its related substances in bulk samples and pharmaceutical dosage forms in the presence of its two process-related impurities. Chromatographic separation was achieved on the reversed phase, Enable column (C18 (5- $\mu \mathrm{m}, 250$ $\times 4.6 \mathrm{~mm}$, i.d.)) at ambient temperature using a mobile phase consisting of $0.02 \mathrm{M}$ potassium dihydrogen orthophosphate (adjusted to $\mathrm{pH} 6$ with $1 \mathrm{M} \mathrm{KOH})$ and acetonitrile $(80: 20$, v/v). Flow rate was $1 \mathrm{~mL} / \mathrm{min}$ and retention time was found to be $7.7 \pm 0.05 \mathrm{~min}$. Quantitation was achieved with UV detection at $215 \mathrm{~nm}$ based on peak area with linear calibration curves at concentration range $75-300 \mu \mathrm{g} / \mathrm{mL}$. Forced degradation studies were performed, in accordance with ICH guidelines, using acidic, alkaline, oxidative, neutral, photolytic, and thermal conditions. The drug was found to be stable under all the conditions. The developed method was validated in terms of precision, robustness, recovery, and limits of detection and quantitation. Regression analysis shows an " $r$ " value (correlation coefficient) of greater than 0.999 for tapentadol and two potential impurities.
\end{abstract}

\section{Introduction}

Chemically tapentadol hydrochloride is 3-[(1R, 2R)-3-(dimethylamino)-1-ethyl-2-methylpropyl] phenol hydrochloride. It is a centrally acting analgesic with dual mode of action as an agonist at the $\mu$-opioid receptor and as a norepinephrine reuptake inhibitor [1]. It is used in the treatment of moderate to severe pain. It was reported that tapentadol dosed at $50 \mathrm{mg}$ 3 times daily for 7 days [2].

Analysis plays an important role in the formulation development of any drug molecule. A suitable and validated method has to be available for the analysis of drugs in bulk, in drug delivery systems, in dissolution studies (in vitro), and in biological samples (in vivo). If such a suitable method for a specific need is not available, then it becomes essential to develop a sensitive, accurate, precise, and reproducible method for the estimation of drug samples.
An extensive literature survey reveals that tapentadol was analyzed in urine sample by the ultra performance liquid chromatography method [3]. A literature survey further revealed the availability of HPLC methods in canine plasma [4] and pharmaceutical formulations $[5,6]$. The computerassisted literature survey reveals that there is no stabilityindicating RP-LC method for the determination of processand degradation-related impurities formed under the stress conditions in tapentadol.

In this paper we describe the development and validation of related substances method for accurate determination of two potential process impurities in tapentadol samples as per the International Conference on Harmonization (ICH) recommendations. The present active pharmaceutical ingredient (API) stability test guideline Q1A (R2) issued by ICH suggests that stress studies should be carried out on active pharmaceutical ingredient (API) to establish its inherent 
<smiles>CCI(c1cccc(O)c1)C(I)C(I)CN(C)CC(I)C(I)C(I)C[N+](C)(C)[O-]</smiles>

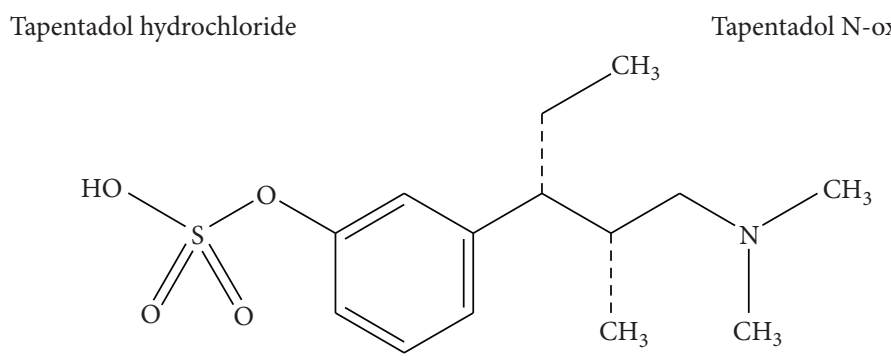

Tapentadol O-sulfate (imp-2)

FIGURE 1: Structure of tapentadol and its process-related impurities.

stability characteristics, leading to separation of degradation products and hence supporting the suitability of the proposed analytical procedures. It also recommends that the analytical test procedures for stability samples should be stability indicating and should be fully validated. Development of an accurate and efficient analytical method for determining the quality and evaluating the impurity profile of drug substances provides some of the critical activities carried out during process research and development in order to meet the requirements of various regulatory authorities $[7,8]$. Stability testing forms an important part of the process of drug product development. The purpose of stability testing is to provide evidence on how the quality of a drug substance varies with time under the influence of a variety of environmental factors such as temperature, humidity, and light, which enables recommendation of storage conditions, retesting periods, and establishing shelf life. Hence a stability-indicating RP-HPLC method was developed for the quantitative determination of tapentadol and its two process-related impurities, namely, imp-1 and imp-2. The present research paper provides a simple, rapid, selective and stability-indicating method for determining the process- and degradation-related impurities in samples of tapentadol bulk drug along with its validation as per the USP and the ICH guidelines $[9,10]$. Here we describe the investigation in detail.

\section{Experimental}

2.1. Materials. Pharmaceutical grade of tapentadol and its related impurities (Figure 1) was kindly supplied by Hetero Drugs., Hyderabad, India, used without further purification, and certified to contain $99.65 \%(\mathrm{w} / \mathrm{w})$ on dried basis. All chemicals and reagents used were of LC grade and were purchased from Qualigens Fine Chemicals (Mumbai, India).
2.2. Instrumentation and Chromatographic Conditions. HPLC method's development and validation were done on a Shimadzu (Tokyo, Japan) liquid chromatograph equipped with an LC-10 AT vp pump, SPD-20A UV detector, Rheodyne $7752 \mathrm{i}$ injector with $20 \mu \mathrm{L}$ loop, and class-vp chromatography software. Peak purity of the various stressed samples was analysed by means of SPD-M10A vp diode array detector. The stationary phase used was an RP-18 Enable column $(5-\mu \mathrm{m}, 250 \times 4.6 \mathrm{~mm}$, i.d. $)$, and the mobile phase used was $0.02 \mathrm{M}$ potassium dihydrogen orthophosphate (adjusted to $\mathrm{pH} 6$ with $1 \mathrm{M} \mathrm{KOH}$ ) and acetonitrile $(80: 20, \mathrm{v} / \mathrm{v})$. The mobile phase was filtered using a $0.45 \mu \mathrm{m}$ membrane filter (Rankem Nylon Membrane, New Delhi, India). High-purity water was prepared by use of a Millipore Milli-Q plus water-purification system. The mobile phase flow rate was $1 \mathrm{~mL} / \mathrm{min}$, and the injection volume was $20 \mu \mathrm{L}$. UV detection was carried out at $215 \mathrm{~nm}$. A degassed mixture of water and acetonitrile in the ratio $(70: 30 \mathrm{v} / \mathrm{v})$ was used for the preparation of standard solutions and test samples and subsequently denoted "diluent." All weighing was done on a Shimadzu electronic balance, BL-220 H.

2.3. Standard Solutions and Calibration Graphs. Standard stock solution was prepared by dissolving $100 \mathrm{mg}$ of tapentadol in $100 \mathrm{~mL}$ diluent $(1000 \mu \mathrm{g} / \mathrm{mL})$. Working solutions were prepared from the above stock solution for related substances and assay determination. A mixed stock solution $(0.3 \mathrm{mg} / \mathrm{mL})$ of the impurities was prepared by weighing $3 \mathrm{mg}$ of imp-1 and imp-2 and made up to $10 \mathrm{~mL}$ with diluent.

2.4. Sample Preparation. To determine the content of tapentadol in conventional tablets (Nucynta: label claim: $100 \mathrm{mg}$ tapentadol per tablet), twenty tablets were weighed, their mean weight determined, and they were finely powdered 
and powder equivalent to $15 \mathrm{mg}$ tapentadol was weighed. Then equivalent weight of the drug was transferred into a $100 \mathrm{~mL}$ volumetric flask containing $50 \mathrm{~mL}$ diluent, sonicated for $30 \mathrm{~min}$, and diluted to $100 \mathrm{~mL}$ with diluent. The resulting solution was centrifuged at $3000 \mathrm{rpm}$ for $15 \mathrm{~min}$. Supernatant was taken, and after suitable dilution, the sample solution was then filtered using $0.45 \mu \mathrm{m}$ filter and the filtrate was used as sample solution.

\subsection{Method Validation}

2.5.1. Precision. Precision of the method was checked by injecting six individual preparations of $300 \mu \mathrm{g} / \mathrm{mL}$ tapentadol spiked with $0.15 \%$ each of imp-1 and imp-2. The RSD (\%) of peak area for imp-1 and imp-2 was calculated. Precision was also determined by performing the sample procedures on a different day (interday precision). The intermediate precision of the method was evaluated when the method was performed by a different analyst using a different column and a different instrument in the same laboratory. The precision of the assay method was evaluated by performing six independent assays of a test sample of tapentadol against qualified reference standard. RSD (\%) was calculated for the six assay values obtained.

2.5.2. Sensitivity. The detection limit of an individual analytical procedure is the lowest amount of analyte in a sample that can be detected but not necessarily quantitated as an exact value. The quantitation limit of an individual analytical procedure is the lowest amount of analyte in a sample that can be quantitatively determined with suitable precision and accuracy. The quantitation limit is a parameter of quantitative assays for low levels of compounds in sample matrices and is used particularly for the determination of impurities and/or degradation products. Sensitivity was determined by establishing limits of detection (LOD) and quantitation (LOQ) for imp-1 and imp-2, estimated as the amounts for which the signal-to-noise ratios were $3: 1$ and $10: 1$, respectively, by injecting a series of dilute solutions of known concentration. Precision was studied at LOQ level by injecting six individual preparations of imp-1 and imp-2 and calculating RSD (\%) of the peaks areas for each impurity.

2.5.3. Accuracy. The accuracy of the assay method was evaluated for five concentrations of the bulk drugs and pharmaceutical dosage forms, that is, $75,125,150,225$, and $300 \mu \mathrm{g} / \mathrm{mL}$. Test solutions were prepared in triplicate and each solution was analysed three times. The bulk sample contained imp-1 at a level of $0.01 \%$ and a total of $0.06 \%$ of unknown impurities (limit: NMT 0.15\% for a single unknown impurity and $0.50 \%$ for total impurities). The study was carried out in triplicate at $0.075,0.125,0.15,0.225$, and $0.3 \%$ of the analyte concentration $(300 \mu \mathrm{g} / \mathrm{mL})$. Percentage recovery for imp-1 and imp-2 was also calculated.

2.5.4. Linearity. Linearity test solutions for the assay method were prepared from stock solution at five concentrations from 50 to $200 \%$ of assay analyte concentration $(75,125,150$,
225 , and $300 \mu \mathrm{g} / \mathrm{mL}$ ). Linearity test solutions for the related substance method were prepared by diluting the impurity stock solution to required concentrations. The solutions were prepared at seven concentrations from the LOQ to $200 \%$ of the permitted maximum level of the impurity (i.e., LOQ, $0.015,0.0375,0.075,0.15,0.225$, and $0.3 \%$ ). The results obtained from chromatography of these solutions were subjected to linear least-squares regression analysis. The calibration equations obtained from regression analysis were used to calculate the corresponding predicted responses.

\subsubsection{Specificity (Forced Degradation Study of Tapentadol).} Specificity is the ability of the method to measure analyte response in the presence of its potential impurities. Stress testing of the drug substance can help to identify likely degradation products, which can, in turn, help to establish the degradation pathways and the intrinsic stability of the molecule and validate the stability-indicating power of the analytical procedures used. All stress degradation studies were performed at an initial drug concentration of $100 \mu \mathrm{g} / \mathrm{mL}$.

Acid and base hydrolysis was performed in $0.5 \mathrm{M} \mathrm{HCl}$ at $60^{\circ} \mathrm{C}$ for 5 days. Basic hydrolysis was performed in $0.5 \mathrm{M}$ $\mathrm{NaOH}$ at $60^{\circ} \mathrm{C}$ for 5 days. For study in neutral solution, the drug was dissolved in water and heated at $80^{\circ} \mathrm{C}$ for 5 days. Oxidation studies were performed at ambient temperature in $3 \%$ hydrogen peroxide for $3 \mathrm{~h}$. Photolytic degradation studies were performed in a Binder KBS240 photolytic chamber in accordance with Option 2 of Q1B in the ICH guidelines. The drug sample was exposed to light for overall illumination of $1.2 \times 10^{6} \mathrm{lux} \mathrm{h}$ and an integrated near-ultraviolet energy of $200 \mathrm{~W} \mathrm{~h} \mathrm{~m}^{2}$. To study thermal degradation a sample of the drug was exposed to dry heat at $80^{\circ} \mathrm{C}$ for 5 days. Samples were withdrawn at appropriate times and subjected to LC analysis to evaluate the capacity of the method to separate tapentadol from its degradation products. Photodiode array detection was used to check and ensure the homogeneity and purity of the tapentadol peak in all the stressed sample solutions.

2.5.6. Robustness of the Method. To evaluate LC method robustness, a few parameters were deliberately varied and the resolution between tapentadol, imp-1, and imp-2 was evaluated. The parameters included variation of C-18 columns from different manufacturers, flow rate, percentage of acetonitrile in the mobile phase, column temperature, and acetonitrile of different lots. Two analytical columns, one (Kromasil C-18 column) from Pune, India, and the other (Finepak C-18 column) from Japan, were used during the experiment.

2.5.7. Solution Stability. The solution stability of tapentadol in the assay method was investigated by leaving sample test solutions in tightly capped volumetric flasks at room temperature for $48 \mathrm{~h}$. The same sample solutions were assayed at $6 \mathrm{~h}$ intervals up to the end of the study period against freshly prepared standard solution. The RSD (\%) of the assay of tapentadol was calculated for the study period during solution-stability experiments. The solution stability of the 

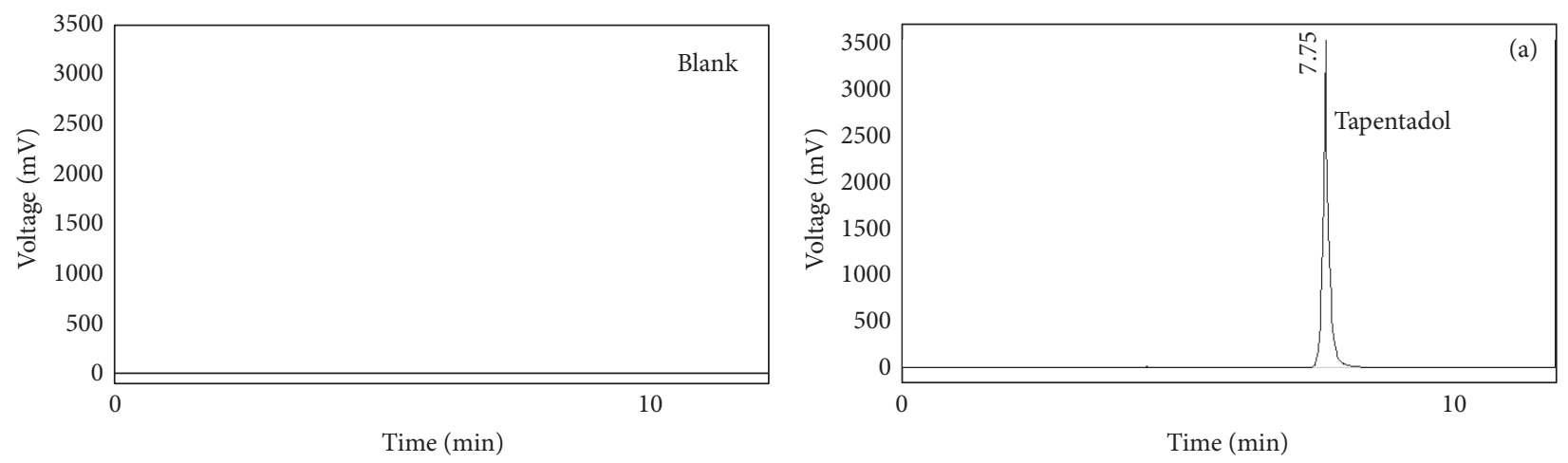

(a)

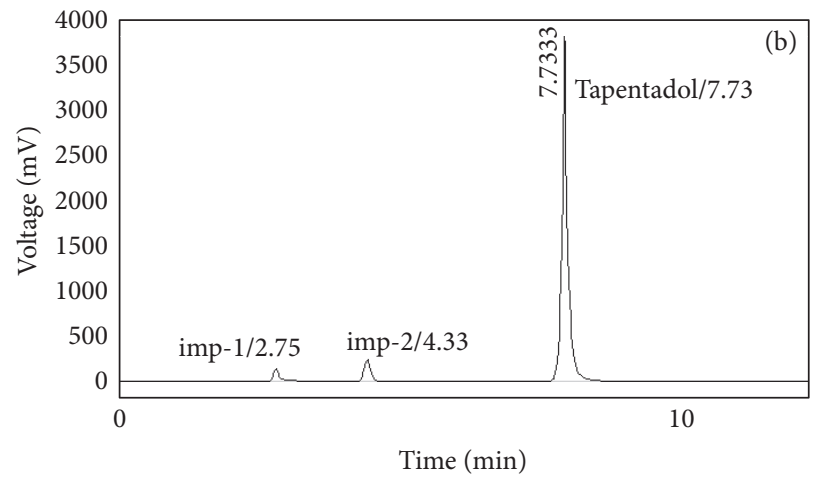

(b)

FIGURE 2: Typical LC chromatograms obtained from the blank (a), tapentadol bulk sample (b), and tapentadol spiked with known impurities.

TABLE 1: System suitability report.

\begin{tabular}{lccc}
\hline Compound & USP resolution $(\mathrm{RS})$ & USP tailing factor $(T)$ & No. of theoretical plates by the USP tangent method $(N)$ \\
\hline imp-1 & - & 1.05 & 4663.5 \\
imp-2 & 6.38 & 1.09 & 33305.9 \\
Tapentadol & 7.39 & 1.16 & 10703.0 \\
\hline
\end{tabular}

related impurities was also studied by leaving a solution of tapentadol spiked with $0.15 \%$ of the impurities in a tightly capped volumetric flask at room temperature for $48 \mathrm{~h}$. The amount of imp-1 and imp- 2 was determined at $6 \mathrm{~h}$ intervals up to the end of the study period.

\section{Results and Discussion}

3.1. Optimization of Procedures. The main target of the chromatographic method is to achieve the separation of impurities (by-products from the synthesis of tapentadol) and the main component tapentadol. Different reverse-phase stationary phases were employed during method development and different kind of mobile phases were studied with combination of acetonitrile. Initially methanol along with water was tried, the drug and its impurities eluted with highly asymmetric peak shapes (USP tailing was more than 2.0), and imp- 2 was highly retained and the retention time of the impurities along with main component was more than 20 minutes. To reduce the run time and to bring about the good resolution between the main component and all the potential impurities, several preliminary chromatographic runs were performed to investigate the suitability for drug content estimation and cost because of the increasing importance of rapid economic analysis in pharmaceutical analysis to increase the throughput. The chromatographic separation of all the impurities was achieved successfully by following the mobile phase of phosphate buffer along with acetonitrile. Ultimately this mobile phase was selected for validation purpose and stability studies. The typical retention time (RT) of tapentadol, imp-1 and 2 are about 7.73, 2.75, and $4.33 \mathrm{~min}$, respectively. The impurities peaks were identified by injecting and comparing with the retention time of the individual compounds. A typical blank chromatogram, a chromatogram of the bulk sample, and a spiked chromatogram of impurities of tapentadol are depicted in Figure 2. The system suitability parameters of tapentadol and its impurities are also shown in Table 1.

3.2. Precision. During study of method precision and intermediate precision for the assay method, RSD (\%) for tapentadol was within $0.3 \%$. In the study of the precision of the 

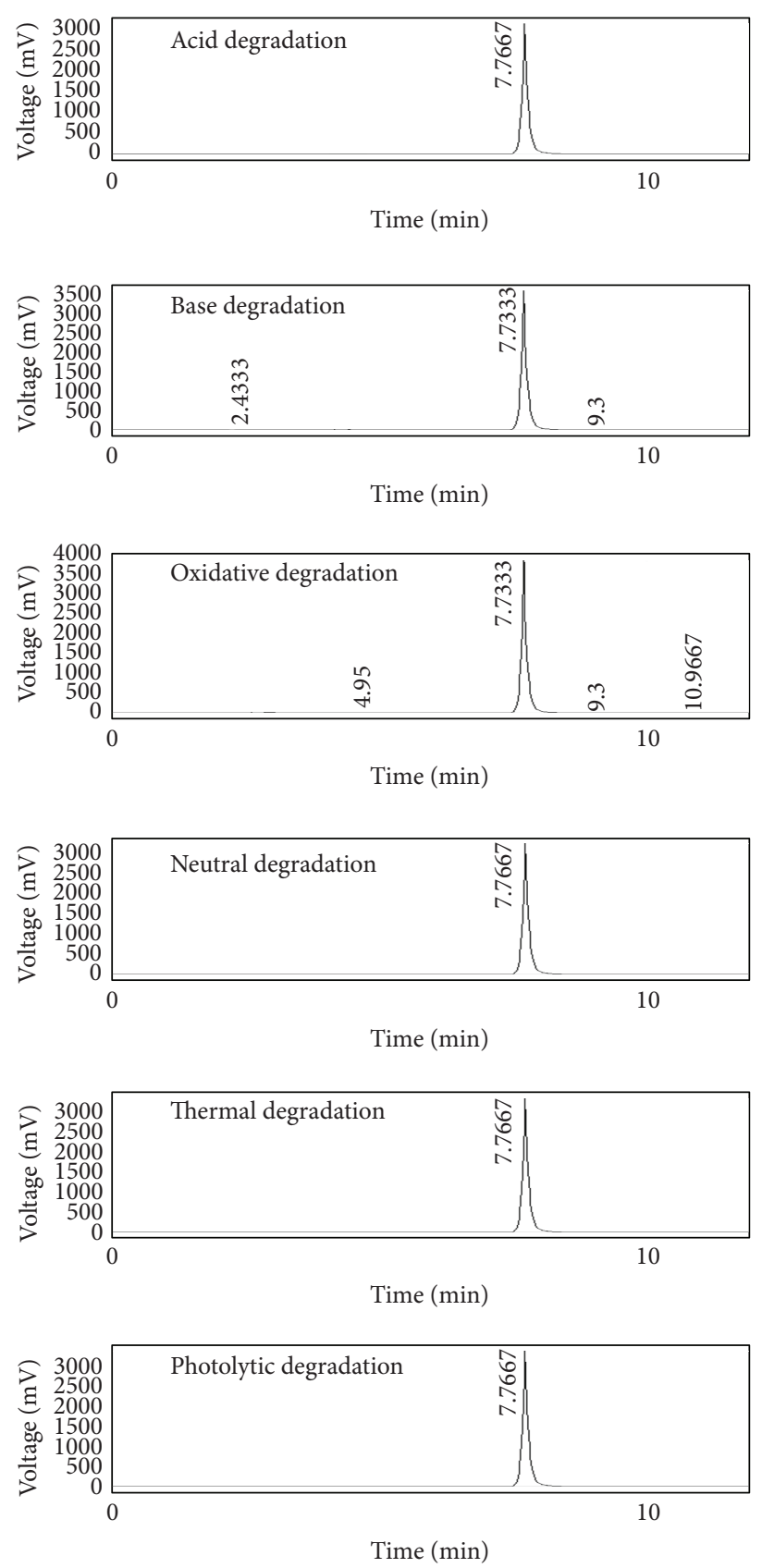

FIGURE 3: HPLC chromatograms of stressed test samples of tapentadol.

TABLE 2: Forced degradation study results.

\begin{tabular}{|c|c|c|c|}
\hline Stress condition & Period of study & $\%$ assay of active substance & $\%$ of degradant \\
\hline Undegraded & - & 99.95 & - \\
\hline Acid hydrolysis $\left(0.5 \mathrm{M} \mathrm{HCl}\right.$, at $\left.60^{\circ} \mathrm{C}\right)$ & 5 days & 99.61 & ND \\
\hline Basic hydrolysis $\left(0.5 \mathrm{M} \mathrm{NaOH}\right.$ at $\left.60^{\circ} \mathrm{C}\right)$ & 5 days & 99.98 & ND \\
\hline Oxidation $\left(3 \% \mathrm{H}_{2} \mathrm{O}_{2}\right.$ at ambient temperature $)$ & $3 \mathrm{hrs}$ & 100.01 & ND \\
\hline Aqueous hydrolysis at $80^{\circ} \mathrm{C}$ & 5 days & 99.20 & ND \\
\hline Thermal degradation & 5 days & 99.63 & ND \\
\hline Photolytic degradation & 11 days & 99.96 & ND \\
\hline
\end{tabular}

ND: not detected. 


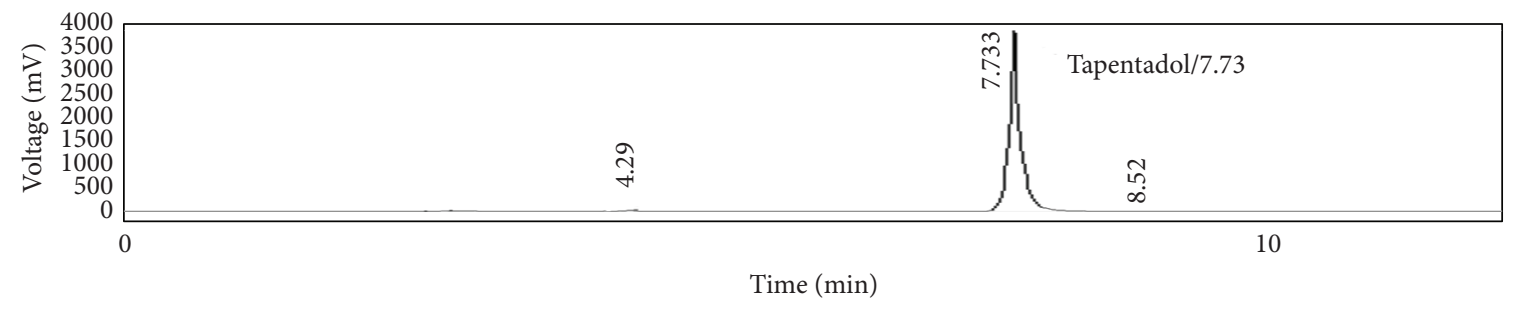

(a) Analysis of formulation 1 where imp-1 is not detected whereas imp-2 and unknown impurity are detected at 4.29 and $8.52 \mathrm{~min}$, respectively

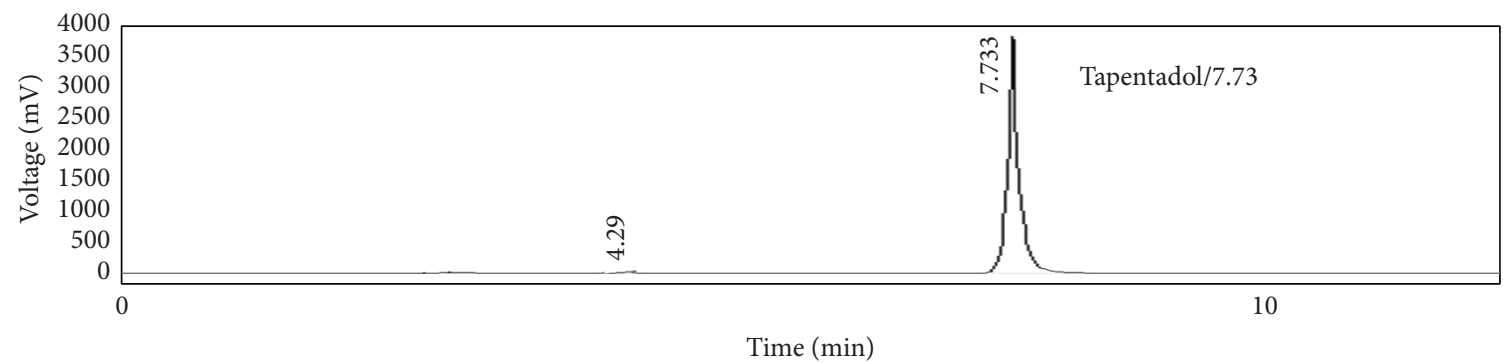

(b) Analysis of formulation 2 where imp-2 is detected at 4.29 min whereas imp-1 is not detected, respectively

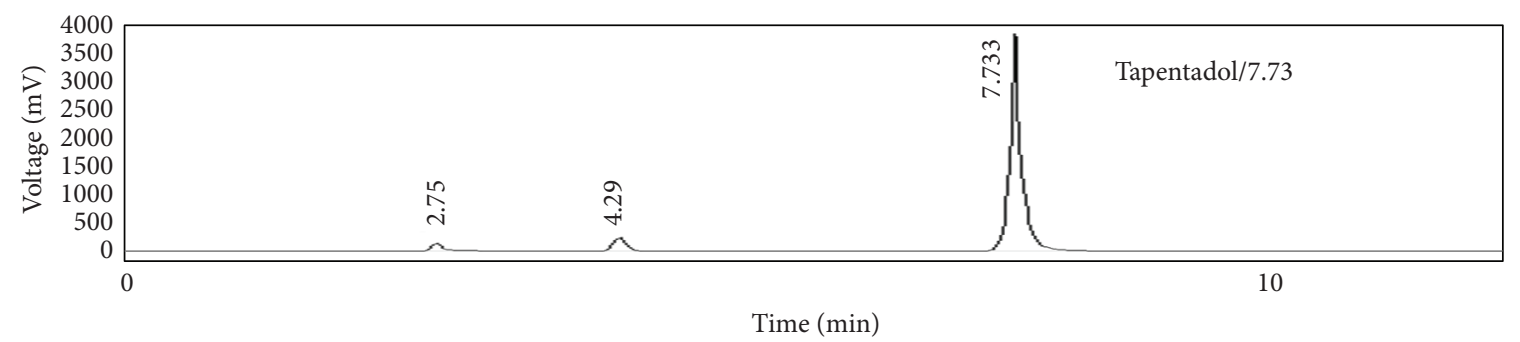

(c) Analysis of bulk sample (B.NO. TAPE P1001) where both imp-1 and 2 are detected

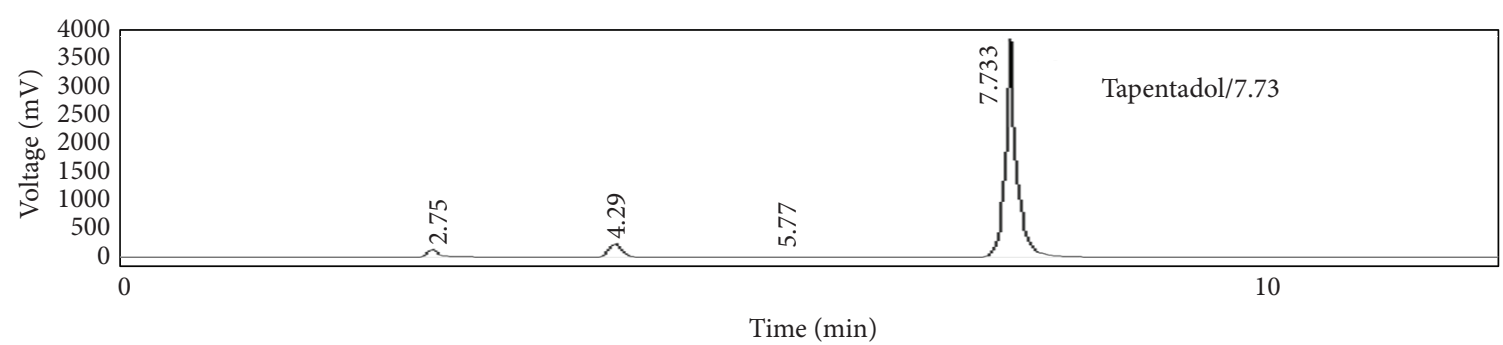

(d) Analysis of bulk sample (B.NO. TAPE P1002) where both imp-1 and 2 are detected. SMUI was detected at 5.77 min, respectively

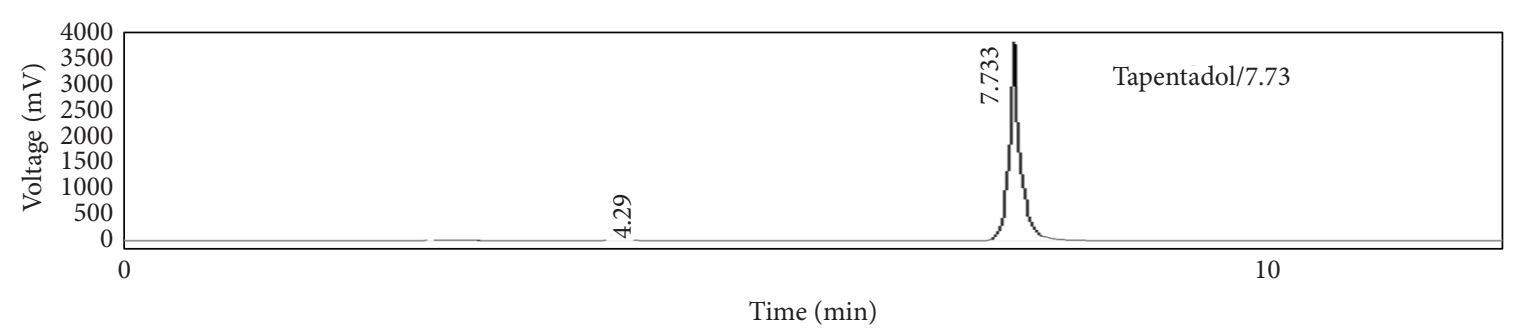

(e) Analysis of bulk sample (B.NO. TAPE P1003) where imp-2 is detected

FIGURE 4: Results (\%) of formulation tablet analysis and bulk drug batch sample analysis. 
TABLE 3: Results (\%) of formulation tablet analysis and bulk drug batch sample analysis.

\begin{tabular}{|c|c|c|c|}
\hline Sample source & imp-1 & imp-2 & SMUI \\
\hline \multicolumn{4}{|c|}{ Formulation product analysis results } \\
\hline Formulation 1 & ND & 0.01 & 0.02 \\
\hline Formulation 2 & ND & 0.01 & 0.07 \\
\hline \multicolumn{4}{|c|}{ Batch analysis results (\%) } \\
\hline B.NO. TAPE P1001 & 0.09 & 0.03 & ND \\
\hline B.NO. TAPE P1002 & 0.02 & 0.02 & 0.05 \\
\hline B.NO. TAPE P1003 & ND & 0.04 & ND \\
\hline
\end{tabular}

ND: not detected; SMUI: single maximum unknown impurity.

related substance method, RSD (\%) of peak area for imp-1 and imp- 2 was within $2 \%$. This confirms the high precision of the method.

3.3. Sensitivity. The limit of detection for imp-1 and imp2 was 0.002 and $0.003 \%$ (of analyte concentration, i.e., $300 \mu \mathrm{g} / \mathrm{mL}$ ), respectively, for $20 \mu \mathrm{L}$ injection volume. Under the same conditions, the LOQ was 0.007 and $0.010 \%$, respectively. Precision for impurities (imp-1 and imp-2) at the LOQ concentrations was $<2 \%$.

3.4. Accuracy. Recovery of tapentadol from bulk samples ranged from 99.3 to $100.6 \%$, and recovery from pharmaceutical dosage forms ranged from 99.2 to $101.3 \%$. Recovery of imp-1 and imp-2 from bulk drug samples ranged from 98.9 to $102.2 \%$.

3.5. Linearity. A linear calibration plot was obtained for the assay method over the calibration range tested, that is, 75-300 $\mu \mathrm{g} / \mathrm{mL}$; the correlation coefficient was $>0.999$. This result is indicative of excellent correlation between the peak area and analyte concentration. In the related substance method linear calibration plots were obtained for imp-1 and imp2 over the calibration ranges tested, that is, from the LOQ to $0.3 \%$; the correlation coefficients were $>0.999$ for all two impurities. The result is indicative of excellent correlation between peak area and concentration for imp-1 and imp-2. When linearity was checked for the related substances over the same concentration ranges on three consecutive days, RSD of the slopes and $Y$-intercepts of the calibration plots were within 2 and 5\%, respectively.

3.6. Results from Forced Degradation Studies. Tapentadol was found to be stable under all the stress conditions like photolytic, acid and base hydrolysis, neutral, thermal and oxidative stress (Figure 3). Peak-purity test results obtained by use of the PDA confirmed that the tapentadol peak was homogeneous and pure, and purity angle was found to be less than that of purity threshold for all the stressed conditioned samples. Assay of tapentadol was unaffected by the presence of imp-1 and imp-2, confirming the stability-indicating power of the method. The result of forced degradation studies of tapentadol was incorporated in Table 2.
3.7. Robustness of the Method. Close study of the analytical results obtained after deliberate alteration of the chromatographic conditions revealed that resolution between impurities and that of the main component were always greater than 2.0, illustrating the robustness of the method.

3.8. Stability in Sample Solution. The RSD of the assay of tapentadol during the solution-stability experiments was within $1.0 \%$. No significant changes in imp-1 and imp-2 content were observed during solution-stability experiments. The results from these experiments confirm that sample solutions used during assay and related substance determinations were stable up to the study period of $48 \mathrm{~h}$.

3.9. Application of the Method. The analysis of commercial formulation sample and bulk drug sample indicated that the method is specific and selective for determination of related substances in the formulation and bulk drug samples (Table 3 and Figure 4). The developed method is capable for quantitative analysis of tapentadol in the bulk drug and in a pharmaceutical dosage form.

\section{Conclusion}

This paper presents a rapid, simple, robust, and accurate RP-LC method that separates the related substances and the degradation products of tapentadol with good resolution. The behaviour of tapentadol under various stress conditions was studied. The method was completely validated to ensure the compliance in accordance with ICH guidelines, and satisfactory results were obtained for all the method characteristics tested. A run time of 10 min enables quick determination of tapentadol and its impurities. Hence, this method can be used for routine testing and stability analysis of tapentadol drug substance, wherein all the statistical results (percentage, RSD, percentage difference, and recovery \%) are within the acceptance criteria. The method would not only be useful for routine evaluation of the quality of tapentadol in bulk drug manufacturing unit but also for detection of impurities in pharmaceutical formulations.

\section{References}

[1] T. M. Tzschentke, T. Christoph, B. Kögel et al., “(-)-(1R,2R)-3(3-dimethylamino-1-ethyl-2-methyl-propyl)-phenol hydrochloride (Tapentadol $\mathrm{HCl}$ ): a novel $\mu$-opioid receptor agonist/ norepinephrine reuptake inhibitor with broad-spectrum analgesic properties," Journal of Pharmacology and Experimental Therapeutics, vol. 323, no. 1, pp. 265-276, 2007.

[2] S. E. Daniels, D. Upmalis, A. Okamoto, C. Lange, and J. Häeussler, "A randomized, double-blind, phase III study comparing multiple doses of tapentadol IR, oxycodone IR, and placebo for postoperative (bunionectomy) pain," Current Medical Research and Opinion, vol. 25, no. 3, pp. 765-776, 2009.

[3] J. A. Bourland, A. A. Collins, S. A. Chester, S. Ramachandran, and R. C. Backer, "Determination of tapentadol (Nucynta) and $\mathrm{N}$-desmethyltapentadol in authentic urine specimens by ultraperformance liquid chromatography- tandem mass spectrometry," Journal of Analytical Toxicology, vol. 34, no. 8, pp. 458-463, 2010 . 
[4] M. Giorgi, A. Meizler, and P. C. Mills, "Quantification of tapentadol in canine plasma by HPLC with spectrofluorimetric detection: development and validation of a new methodology," Journal of Pharmaceutical and Biomedical Analysis, vol. 67-68, pp. 148-153, 2012.

[5] R. P. Kanzariya, T. P. Patel, K. G. Kapuriya, and S. D. Faldu, "Method development and validation of tapentadol hydrochloride by RP-HPLC method," Inventi Impact: Pharm Analysis \& Quality Assurance. In press.

[6] J. Gandhi, N. J. Shah, and A. N. Lumbhani, "Simple, rapid and cost effective method for routine analysis of tapentadol hydrochloride: a novel analgesic drug in bulk and pharmaceutical dosage form by RP-HPLC," Pharma Science Monitor. In press.

[7] International Federation of Pharmaceutical Manufacturers and Associations (IFPMA), "Impurities in new drug substances," in Proceedings of the International Conference on Harmonization (ICH '06), Methodology Q3A(R2), Geneva, Switzerland, 2006.

[8] International Federation of Pharmaceutical Manufacturers and Associations (IFPMA), "Good manufacturing practice guide for active pharmaceutical ingredients," in Proceedings of the International Conference on Harmonization (ICH'05), Methodology Q7A, Geneva, Switzerland, 2005.

[9] The United States Pharmacopeia, Validation of Compendial Methods, United States Pharmacopeia, 32nd edition, 2009.

[10] International Federation of Pharmaceutical Manufacturers and Associations (IFPMA), "Validation of analytical procedures," in Proceedings of the International Conference on Harmonization (ICH '96), Methodology Q2 (R1), Geneva, Switzerland, 1996. 

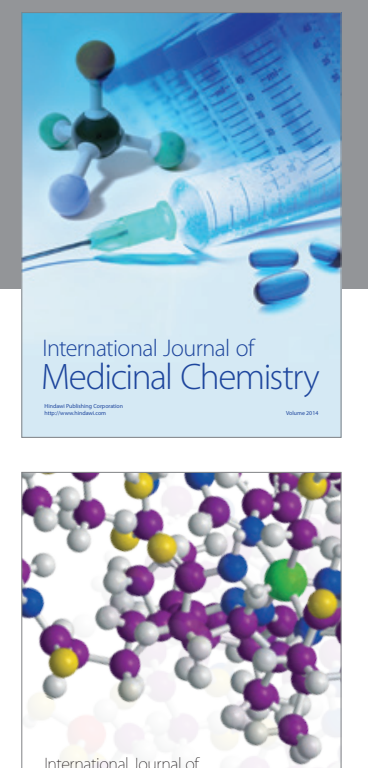

\section{Carbohydrate} Chemistry

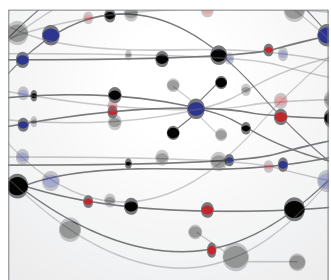

The Scientific World Journal
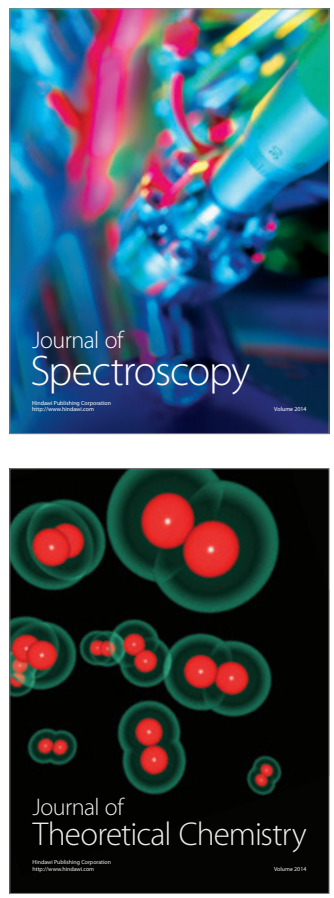
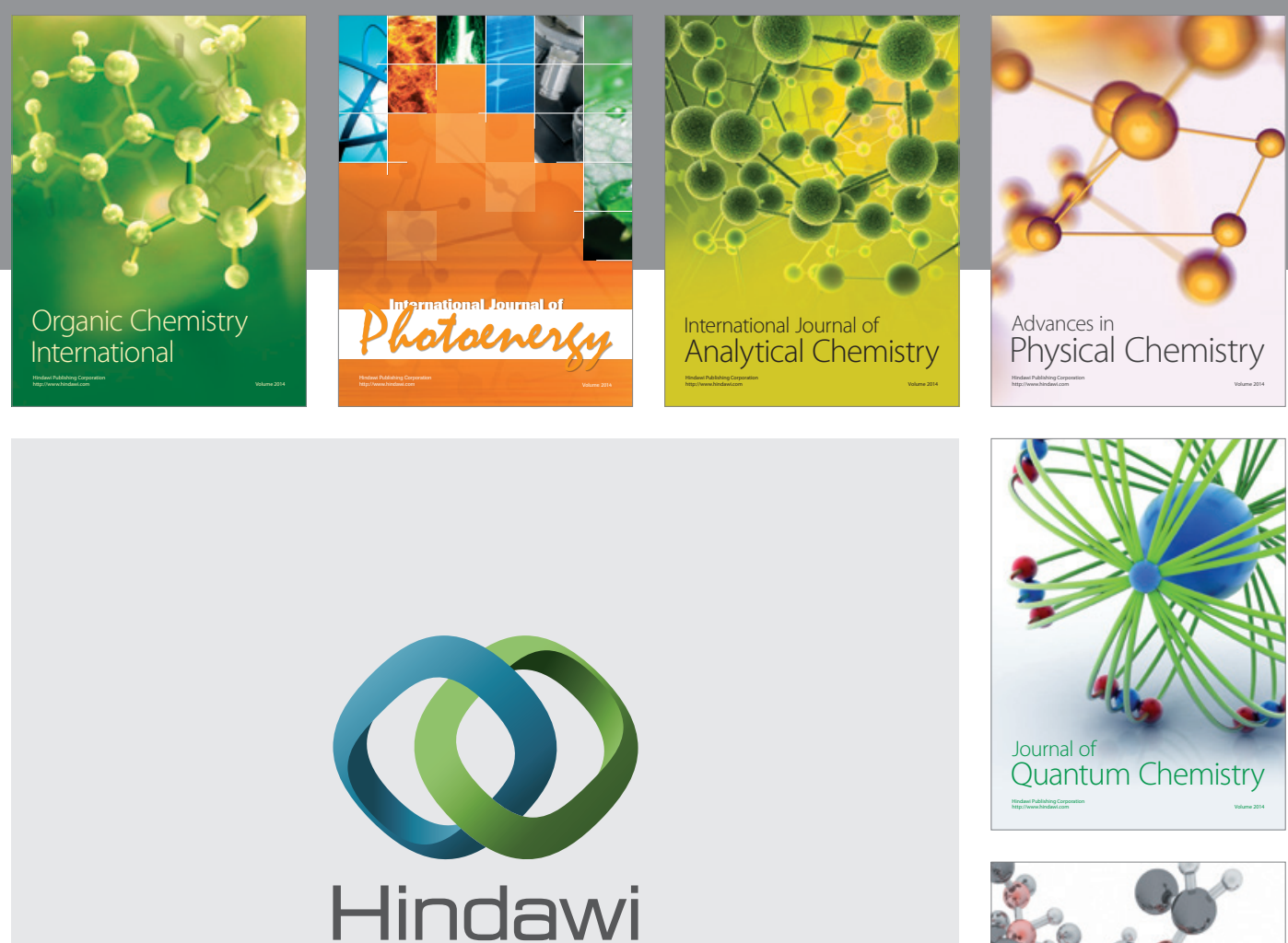

Submit your manuscripts at

http://www.hindawi.com

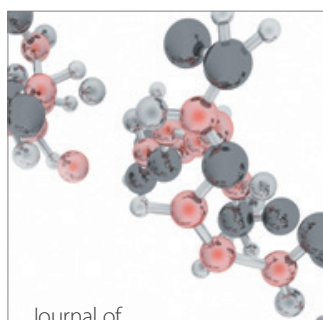

Analytical Methods

in Chemistry

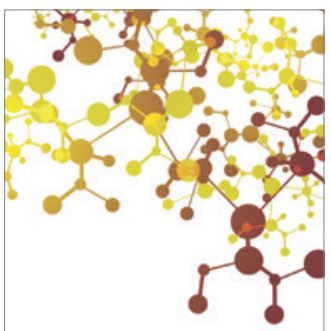

Journal of

Applied Chemistry

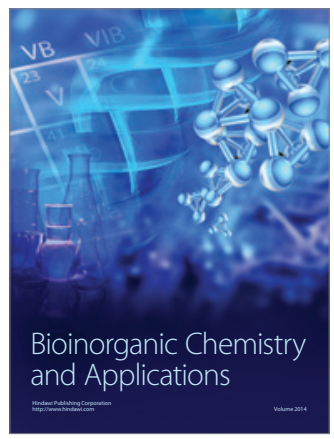

Inorganic Chemistry
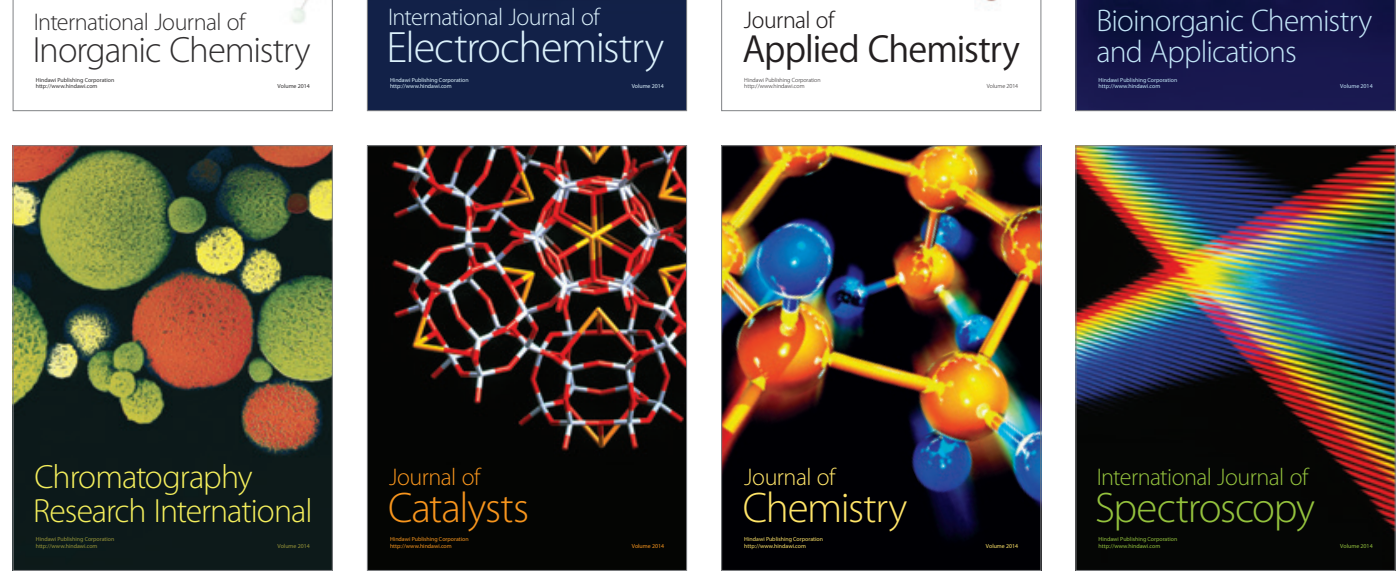